\title{
Analysis of Heat IIIness using Michigan Emergency Department Syndromic Surveillance
}

\author{
Fatema Mamou* and Tiffany Henderson \\ Michigan Department of Community Health, Lansing, MI, USA
}

\section{Objective}

The purpose of this work was to conduct an enhanced analysis of heat illness during a heat wave using Michigan's Emergency Department Syndromic Surveillance System (MSSS) that could be provided to Public Health and Preparedness Stakeholders for situational awareness.

\section{Introduction}

The MSSS, described elsewhere (1), has been in use since 2003 and records Emergency Department (ED) chief complaint data along with the patient's age, gender and zip code in real time. There were 85/139 hospital EDs enrolled in MSSS as of June 2012, capturing $77 \%$ of the annual hospital ED visits in Michigan. The MSSS is used routinely during the influenza season for situational awareness and is monitored throughout the year for aberrations that may indicate an outbreak, emerging disease or act of bioterrorism. The system has also been used to identify heat-related illnesses during periods of extreme heat. Very young children, the elderly, and people with mental illness and chronic diseases are at the highest risk of preventable heatrelated illnesses including sunburn, heat exhaustion, heat stroke and/or death (2). During a heat wave in the summer of 2012, data was reviewed on an ad hoc basis to monitor potential increases in heat-related ED visits.

\section{Methods}

MSSS ED visits were queried to identify those with the primary complaints of: "heat", "sun", or "dehydration" including word derivatives and misspellings. The query excluded terms and misspellings such as "Sunday", "heater", and "heatlh". Daily maximum temperatures for four major cities in Michigan were tracked using measures from the National Oceanic and Atmospheric Administration's National Weather Service (3). Multiple analyses were performed. For this abstract, ED data from a 10-day period of sustained above normal temperatures are presented with data from the prior 10day period used as reference.

Visits were categorized into 1 of 3 syndromes based on the chief complaint: sun-associated, heat-associated, and dehydration. Gender, age group, and syndrome for the period of interest were compared to the reference period. Heat-related visits during the period of extreme heat were also analyzed by Michigan Public Health Preparedness Region.

\section{Results}

During the period of June 28-July 7, 2012 the South and Central regions of Michigan sustained maximum daily temperatures surpassing $90^{\circ} \mathrm{F}$ with maximum temperatures at or above $100^{\circ} \mathrm{F}$ on at least 2 days. Among the cities reviewed, a total of 9 high temperature records were set or tied during that period. The number of heat-related ED visits reported into MSSS increased compared to the previous period of June 18-June 27, 2012. Heat-associated ED visits such as heat exhaustion and heat stroke were more frequent than the reference period, $30.0 \%$ vs. $13.7 \%(\mathrm{p}<0.0001)$. Sun-associated ED visits such as sunburn were lower compared to the reference period, $17.3 \%$ vs. $23.8 \%(\mathrm{p}=0.01)$. Dehydration complaints were elevated among those $20-29$ years of age, $17.7 \%$ vs. $10.0 \%(\mathrm{p}=0.01)$. While the proportion of ED visits due to heat-related complaints was highest in the Central and Northwestern areas of the state, increases were observed in all regions of Michigan.

On July 6, 2012 an initial analysis summary was issued via the Michigan Health Alert Network (MIHAN) to provide situational awareness related to a concurrent heat advisory for much of the state. By July 23, $2012 \mathrm{MDCH}$ issued a media release reporting this increase in heat-related ED visits.

\section{Conclusions}

Although cases used in the analysis may not represent all potential cases of heat-related illness and also may represent non-heatrelated illnesses, ED data are useful in describing trends in illness presentations over time. As the MSSS covers a large proportion of Michigan's population, the data from the MSSS can be stratified by type of heat-related injury, age group, and region, providing detailed situational awareness to public health stakeholders. This type of indepth analysis further contributes to our knowledge of heat events and allows public health to relay important information regarding the severity of the situation and information about groups at risk for illness.

\section{Keywords}

syndromic surveillance; heat illness; extreme heat

\section{References}

1. Sheline KD. Evaluation of the Michigan Emergency Department Syndromic Surveillance System. Advances in Disease Surveillance. 2007; 4: 265

2. CDC. Extreme Heat Prevention Guide. 2012. Available from: http://emergency.cdc.gov/disasters/extremeheat/heat_guide.asp

3. National Oceanic and Atmospheric Administration's National Weather Service. Available from: http://www.nws.noaa.gov/climate/ 